\title{
A case of chimerism-induced paternity confusion: what ART practitioners can do to prevent future calamity for families
}

\author{
Kayla M. Sheets ${ }^{1}$ (D) $\cdot$ Michael L. Baird $^{2} \cdot$ Julie Heinig $^{2} \cdot$ Debra Davis $^{2} \cdot$ Mary Sabatini $^{3}$. \\ D. Barry Starr ${ }^{4}$
}

Received: 13 June 2017 / Accepted: 5 October 2017 /Published online: 23 October 2017

(C) The Author(s) 2017. This article is an open access publication

\begin{abstract}
In the fertility clinic setting, a negative DNA paternity test result usually suggests a sample mix-up likely occurred at the testing company or in the clinic. However, we report a case where, despite repeat negative paternity test results, the alleged father (referred to as "the proband") was confirmed to be the baby's father. The proband, a 34 yearold male, contacted our research group when routine blood testing revealed discrepant blood types between the parents and the baby, repeat paternity tests were negative (excluding the proband as the baby's father), and the fertility clinic found no evidence of any wrongdoing. Microarray technology was utilized to confirm biological relatedness, which revealed an avuncular (uncle/nephew) relationship. Additional tissue samples were analyzed and family studies were conducted at paternity and forensic laboratories using STR-based DNA tests to elucidate the proband's condition of congenital tetragametic chimerism. His paternity was subsequently affirmed and the fertility clinic exonerated of claims of a semen sample mix-up. This case underscores the possibility that
\end{abstract}

Electronic supplementary material The online version of this article (https://doi.org/10.1007/s10815-017-1064-6) contains supplementary material, which is available to authorized users.

Kayla M. Sheets

kayla@ vibrantgene.com

1 Vibrant Gene Consulting, LLC, PO BOX 390752, Cambridge, MA 02139, USA

2 DNA Diagnostics Center, Inc., One DDC Way, Fairfield, OH 45014 , USA

3 Reproductive Endocrinology and Fertility, Massachusetts General Hospital, 55 Fruit St, Boston, MA 02114, USA

4 Department of Genetics, Stanford University School of Medicine, Stanford, CA 94305-5120, USA some allegations of fertility clinic missteps may be explained by undiagnosed chimerism, a condition where an individual harbors two distinct genomes. We offer specific suggestions for improving laboratory reporting and creating clinical guidelines to aid in identifying and rectifying future cases of false exclusions of paternity due to chimerism.

Keywords Congenital chimera - Tetragametic chimerism . Semen sample mix-up · Gamete mix-up · Assisted reproductive technology $\cdot$ False negative DNA paternity testing

\section{Introduction}

Parental (paternity/maternity) DNA testing is indicated where a parent-child relationship requires confirmation. Common reasons for testing are child custody, child support, and immigration cases. In the USA, well over 382,000 legal paternity tests are ordered annually. (With less than $60 \%$ of all surveyed paternity testing laboratories reporting data to the American Association of Blood Banks [AABB] in 2010, this is a considerable underestimate.) Additionally, an unknown number are self-administered at home.

Currently, the gold standard technology used for these tests is a PCR-based assay that amplifies short tandem repeat (STR) regions within the tested person's DNA [1]. This technology was developed in the 1980s and has known limitations and failures [2]. Understanding these is of critical importance. A negative result, where the proband is excluded as the father of the child, has serious consequences to families ranging from broken trust (fidelity concerns), divorce, lost child support, or denied immigration. In cases where a baby was conceived in a fertility clinic, false negative tests can have ramifications for the clinic and staff. 
In this paper, we present a case where the gold standard STR-based DNA paternity test repeatedly excluded the proband from being the biological father, despite the fact that the child was conceived by a semen sample provided by the proband. Unbeknownst to the family and to the fertility clinic, the reason for the test's false negative result was that the father harbored more than one genome, a condition called chimerism.

The specific form of congenital chimerism in our case study was determined to be tetragametic chimerism (tetra = four gametes). Such chimerism can occur when cells from dizygotic twin embryos fuse early in development leading to the birth of a singleton with two cell lines containing two different genomes [3]. If a male with tetragametic chimerism conceives a child with a sperm cell that contains a genome distinct from that of the tested paternal tissue (usually buccal or peripheral blood), standard paternity DNA tests will report non-paternity. Through follow-up DNA testing, the genetic relationship between the proband and the child can be more accurately depicted as avuncular. After all, the DNA of the proband's tested tissue is more similar to the child's uncle, not father.

The Guidance for Standards for Relationship Testing Laboratories (published by the Relationship Testing Program Unit of the American Association of Blood Banks) currently does not have provisions that look for avuncular relationships in standard PCR-based paternity tests. Therefore, current guidance does not safeguard against false negative results for chimeric parents. Moreover, since chimerism poses little or no threat to the individual's health, and since most chimeras do not display associated traits, the majority of existing chimeras are believed to be undiagnosed [3, 4]. As a result, an unknown number of chimeras are at risk of being falsely excluded from being the biological parents of their children by the current gold standard PCR-based paternity tests.

Below, we present the details of the studied case. To the best of our knowledge, this is the first confirmed case of a father with tetragametic chimerism having a child with the genome originating from his unborn twin. In other words, this is the first confirmed case of a man fathering his unborn brother's child and so, in essence, fathering his nephew.

We suggest improvements to current laboratory standards that take into account chimerism and reduce the risk of false paternity exclusions for chimeric individuals. We provide clinical suggestions for assisted reproductive technology (ART) practitioners, in the event of accusations of mistakes and wrongdoing. We provide clinical guidelines for when to suspect undiagnosed chimerism as a cause of purported false relationship DNA test results, and when to consider contacting a genetic expert.

\section{About chimerism}

Chimerism is a condition where an individual carries more than one complete genome. Chimerism can be acquired or congenital. Acquired chimerism can arise in multiple ways.
Low levels of chimerism can be acquired via blood transfusions, or bone marrow or organ transplants, when cells from the donor become incorporated in the recipient's body. In women, acquired chimerism has been detected after pregnancy, when embryonic cells from the fetus circulate and colonize tissues of the mother's body. Maternal-fetal microchimerism may in fact be the most common form of chimerism found in humans $[5,6]$. Such chimerism could occur even if the pregnancy did not survive to recognition [3].

Congenital chimerism can arise through various mechanisms. Blood-exclusive chimeras can result from blood vessel anastomoses between dichorionic placentas. Extensive testing of additional tissues is required to diagnose patients with chimerism exclusive to blood. This testing is often not feasible, leading to speculation that these individuals may have additional tissues involved. [7] Fertilization of an egg and associated polar body by two sperm, followed by their subsequent fusion can lead to chimerism. Tetragametic chimerism occurs when cells from dizygotic twin embryos fuse early in development leading to the birth of a singleton with two cell lines containing two different genomes.

This case study involves diagnosing an individual with tetragametic chimerism, who unknowingly had what we refer to as a major and minor genome. The major genome is defined as the chimera's predominant genome, while the minor genome is defined as the less predominant genome which presumably originated from the DZ twin.

\section{Prevalence of tetragametic chimerism in the era of fertility treatments}

The prevalence of tetragametic chimerism remains largely unknown. Most tetragametic chimeras do not display any associated traits. Moreover, no public-wide screening program exists to diagnose chimerism since it poses little risk to the individual's health. As a result, the majority of existing tetragametic chimeras remain undiagnosed throughout their lifetime, which makes the prevalence of their condition unknown.

Well-established data indicates that one in eight singleton pregnancies originated as a dizygotic twin pregnancy with no evidence of a twin history [8]. It is possible this figure is an underestimate due to the increased use of assisted reproductive technologies. Such pregnancies have the chance of resulting in tetragametic chimeras, but no data exists how frequently this occurs.

Various methods employed by ART are known to increase the rate of multiple pregnancies (e.g., mono and dizygotic twins, triplets). Dizygotic multiple pregnancies are believed to be the leading cause of tetragametic chimerism. ART can also increase the likelihood of monochorionic dizygotic twinning (MC DZT) [9], with five such cases reported in Japan in 2004 alone [10]. Notably, this finding was reported to be an 
underestimate of the prevalence of MC DZT cases caused by ART. These cases were detected because of sex chromosome discordance discovered in blood samples (thought to be confined blood chimerism). Thus, the cases involving concordant sex chromosomes were missed.

\section{Results}

\section{Methods summary}

\section{Ancestry DNA testing}

Kits were ordered for the presumed genetic father (proband) and child from 23 andMe which utilizes the Illumina HumanOmniExpress-24 format chip. The biological relationship was assessed using the relationship finder application.

\section{Confirmatory paternity and forensic DNA testing}

Buccal samples were obtained from the mother, child, and proband as well as from the parents of the proband. At DNA diagnostic center (DDC), DNA was extracted from the buccal swabs using organic extraction. Additionally, a semen sample, a blood sample, and skin swabs were collected from the alleged father and DNA was isolated using a differential extraction method. DNA analysis was performed using an Applied Biosystems AmpFlSTR Identifiler® PCR Amplification Kit, a 310 genetic analyzer and Gene Mapper ID v 3.2. The forensic lab was asked to analyze blood and semen samples as they use the more sensitive genetic analyzer, an $\mathrm{ABI} 310$, as opposed to the ABI 3730 used by the paternity testing lab. All other samples were analyzed in DDC's paternity testing lab.

Our group received the proband's formal consent to publish his case.

\section{Identifying the avuncular relationship}

The proband, a 34 year-old Caucasian male, presented to our group and later revealed he had patchy bi-toned skin. He and his wife were diagnosed with idiopathic infertility and reported no clinical history of therapeutic transplantation or transfusion. The couple conceived their second son via intrauterine insemination (IUI). At birth, the baby's blood type (AB+) was found to be inconsistent with the blood types of the proband and his wife (A+ and $\mathrm{A}^{-}$, respectively). The couple contacted the fertility clinic with concerns of a possible mistake. The clinic launched an internal investigation and concluded the proband was the sole Caucasian semen donor that day.

Seeking answers, the couple pursued at-home DNA paternity testing. This testing utilized PCR amplification of the standard 15 STR markers from buccal-derived DNA samples. The test determined that 12 of 15 markers matched, but yielded a probability of paternity of $0 \%$, excluding the proband from being the baby's father.

Because of concerns that the lab or clinic may have made an error, the couple contacted an attorney, who facilitated the procurement of a legal paternity test. The second test, conducted at a different lab, concluded that 11 of 15 alleles matched, but again excluded the proband from being the biological father of the child. Both labs queried the same STR markers and differed in the number that matched, and neither paternity test gave any indication of the possibility of a second degree or more distant biological relationship between the proband and the child.

The senior author (Starr) was contacted, and at his recommendation, the proband and child pursued relationship DNA testing that utilized single nucleotide polymorphism (SNP) microarray technology to determine if there was a biological relationship that had been overlooked. Testing was performed by 23 andMe, using the Illumina HumanOmniExpress-24 chip, querying over 700,000 markers. This testing and subsequent use of the relative finder feature identified an avuncular (uncle/nephew) relationship, demonstrating $\sim 25 \%$ shared DNA homology (Fig. 1) between the proband and child. This led Starr to suspect that the proband could be a chimera.

The primary author (Sheets) recommended confirmatory testing for the proband and facilitated testing at DNA Diagnostics Center (DDC). The proband provided several different tissue samples and proceeded with testing buccal samples from his wife, his parents, and his two sons (Table 1).

Elucidating the cause of the proband's false negative standard DNA paternity tests and avuncular relationship to his son required confirmatory testing and custom laboratory interpretations. First, the proband's additional tissue samples were tested to confirm the suspected diagnosis of chimerism. Next, confirmatory testing was required to identify the origin of the proband's additional alleles. Last, the laboratory devised an algorithm to calculate the probability of paternity using the proband's minor genome.

\section{Confirmatory testing: diagnosing the proband}

DDC extracted DNA from each of the proband's tissue samples (blood, semen, hair, skin, and buccal). Two specialized laboratories at DDC were used to analyze the samples via STR-based assays, a paternity lab and a forensic lab. Paternity testing was conducted on all samples. Additional forensic testing was performed where the paternity testing was inconclusive.

In most samples, the paternity lab detected the presence of one of the following: a major genome, a minor genome, or two genomes (Table S1). Again, we define the major genome as the chimera's predominant genome and the minor genome as the less predominant genome (which presumably originated 


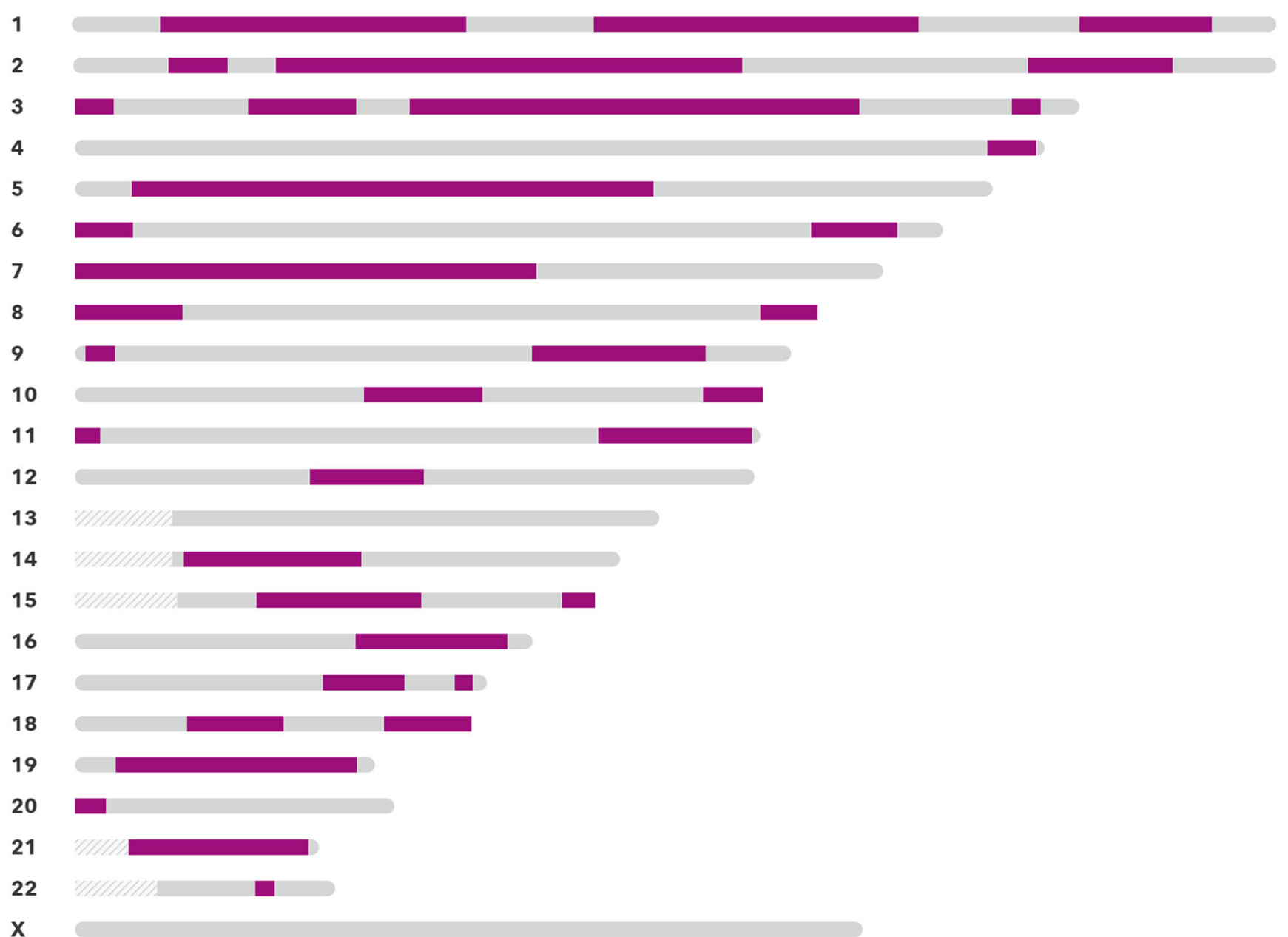

Fig. 1 At-home DNA relationship testing result. Ancestry test result depicts chromosomes, shown by gray bars, and ordered according to autosomal number. The green regions represent segments of contiguous SNPs that are shared between the proband and child. In a typical parent/

Table 1 Proband's familial studies. The proband's immediate family members provided buccal samples for STR-based DNA testing at DDC's relationship testing laboratory. The major genome is defined as the chimera's predominant genome, while the minor genome is defined as the less predominant genome, which presumably originated from the DZ twin. The first son's DNA testing revealed the presence of the proband's major genome. Meanwhile proband's second son's DNA showed the presence of the minor genome and correlated to the proband's semenderived DNA sample

\begin{tabular}{|c|c|c|c|}
\hline Relatives & Tissue type & $\begin{array}{l}\text { DNA test } \\
\text { method }\end{array}$ & Result \\
\hline Wife & Buccal & 15 STR & All alleles accounted for in sons \\
\hline 1 st son & Buccal & 15 STR & Major genome \\
\hline 2nd son & Buccal & $15 \mathrm{STR}$ & Minor genome \\
\hline $\begin{array}{l}\text { Proband's } \\
\text { father }\end{array}$ & Buccal & 15 STR & $\begin{array}{l}\text { All alleles accounted for in major } \\
\text { and minor genome of proband }\end{array}$ \\
\hline $\begin{array}{r}\text { Proband's } \\
\text { mother }\end{array}$ & Buccal & $15 \mathrm{STR}$ & $\begin{array}{l}\text { All alleles accounted for in major } \\
\text { and minor genome of proband }\end{array}$ \\
\hline
\end{tabular}

child relationship, the green regions would have longer contiguous green lines across the length of chromosomal segments, rather than short green lines that represent the $\sim 25 \%$ of shared DNA, as is seen in this uncle/ nephew biological relationship from the DZ twin.) The blood and semen samples required testing in the forensic lab as the standard paternity testing assays failed due to the limited amount of DNA present.

This testing successfully identified two distinct cell lines in the proband's semen. DDC estimated that the minor genome, the source of the DNA for the proband's second child, comprised about $10 \%$ of the semen sample. Forensic testing was able to detect only the major genome in the proband's blood, though evidence suggested that a second genome may be present. A recent blood typing analysis revealed the proband has predominantly $\mathrm{A}+$ but also detected $\mathrm{AB}$ blood, indicating it is likely both genomes are present in blood. Presumably, this recent serum test was a more sensitive analysis than what had been used in prior years, implementing forward and back typing.

The proband's buccal sample was evaluated in several commercial laboratories, using different DNA testing methodologies (Table 2). Standard STR testing at two paternity 
Table 2 Comparing methodologies: testing outcomes of Proband's Buccal DNA. The proband's buccal-derived DNA sample was analyzed with three different molecular genetic testing platforms. Each methodology yielded varying conclusions. Standard STR-based analysis in paternity laboratories excluded the proband from being the child's father, providing no indication of a more distant blood relationship. The forensic laboratory utilized a more sensitive protocol, and only detected the proband's major genome. The SNP-based array used by the ancestry testing laboratory identified a second-degree (avuncular) relationship between the proband and child

\begin{tabular}{lll}
\hline $\begin{array}{l}\text { Paternity testing } \\
(15 \text { STRs })\end{array}$ & $\begin{array}{l}\text { Forensic testing } \\
(15 \text { STRs })\end{array}$ & $\begin{array}{l}\text { Ancestry testing } \\
\text { (SNPs) }\end{array}$ \\
\hline Exclusion & $\begin{array}{l}\text { Major genome } \\
\text { detected }\end{array}$ & $\begin{array}{c}\text { Second-degree } \\
\text { relationship }\end{array}$ \\
\hline
\end{tabular}

laboratories (Genex Diagnostics, DDC) did not detect the proband's minor genome. STR testing in a forensic laboratory (DDC) also only detected the major genome. Testing at an ancestry laboratory (23andMe) did not detect the minor genome in the proband but elucidated a second-degree relationship with the proband's second child.

Overall, the confirmatory testing at DDC ascertained that the proband was a chimera, whose sperm, among other tissues, contained two distinct genomes.

\section{Confirmatory testing: family members}

Further testing was conducted to better understand the origin of the proband's alleles and how they could be used to investigate his paternity.

DNA from the proband's wife was used to identify maternally inherited alleles in his second child. Through the process of elimination, this allowed the identification of alleles that this son likely inherited from the proband's minor genome. This data helped enhance the accuracy of the subsequent paternity test probability estimation and interpretation.

DNA samples from the proband's parents were used as controls. Each allele in the proband needed to be accounted for in his mother and father (Figure S1 and Table S2). This study confirmed that no external DNA had contaminated the proband's tissue samples, further strengthening the conclusion that the proband contained two closely related genomes.

\section{Confirmatory testing: resolving the proband's paternity}

DDC confirmed paternity of the proband for both of his sons using paternity and forensic DNA tests. Paternity testing of buccal-derived DNA from the proband and his first son did not exclude the proband as the father (data not provided).

Table 3 Forensic DNA testing of proband's semen confirms paternity of the second son DDC DNA test report. The proband's semen contains two cell lines with multiple alleles present at each locus. The combined paternity index (CPI) of 134,323 was calculated using minor alleles

\begin{tabular}{|c|c|c|c|c|c|c|c|c|c|}
\hline & & Mot & lccal) & Chil & cal) & Alle & ther ( & & \\
\hline Locus & PI & Alle & & Alle & & Alle & & & \\
\hline D3S1358 & 1.88 & 15 & 16 & 15 & & & 15 & 18 & \\
\hline vWA & 1.21 & 18 & 19 & 17 & 19 & 16 & 17 & 19 & \\
\hline D16S539 & 1.41 & 12 & 13 & 12 & 13 & 11 & 12 & 13 & \\
\hline CSF1PO & 1.10 & 11 & 12 & 11 & & 10 & 11 & 12 & \\
\hline TPOX & 0.62 & 8 & 9 & 8 & & 8 & 9 & 11 & \\
\hline D8S1179 & 3.36 & 10 & 14 & 10 & 15 & 11 & 13 & 15 & \\
\hline D21S11 & 10.79 & 29 & 33.2 & 27 & 33.2 & 27 & 29 & 33.2 & \\
\hline D18S51 & 5.88 & 15 & 17 & 15 & 19 & 15 & 16 & 19 & 22 \\
\hline D19S433 & 1.70 & 13 & 14 & 13 & 14 & & 13 & 14 & \\
\hline TH01 & 1.64 & 6 & 8 & 6 & 9.3 & & 6 & 9.3 & \\
\hline FGA & 2.36 & 20 & 24 & 20 & 24 & 20 & 23 & 24 & \\
\hline D5S818 & 1.37 & 11 & 12 & 11 & 12 & & 11 & 12 & \\
\hline D13S317 & 4.47 & 8 & 12 & 8 & 13 & & 12 & 13 & \\
\hline D7S820 & 2.41 & 9 & 10 & 9 & 11 & & 9 & 11 & \\
\hline D2S1338 & 2.98 & 19 & & 19 & & 17 & 19 & 22 & \\
\hline Amelogenin & & $X$ & & $X$ & Y & & $X$ & $\mathrm{Y}$ & \\
\hline Interpretation: & & & & & & & & & \\
\hline Combined paternity index: & 134,323 & & & Prol & of pa & 99.9 & & & \\
\hline
\end{tabular}


Forensic testing of the proband's semen and his second son's buccal sample did not exclude the proband as the father (Table 3). This was consistent with the hypothesis that the proband had tetragametic chimerism and had fathered each son with a different genome.

\section{Families with chimerism present complex relationships}

This case illustrates the unusual relationships that arise within families where one parent has tetragametic chimerism (Fig. 2). The proband's second child was conceived via sperm that contained the proband's minor genome, which originated from the proband's unborn twin (II.3). As a result, the proband (based on his major genome) is a second degree relative to this son (III.2), thus they are more similarly related as an uncle/nephew. However, based on his minor genome, the proband is also the father. The two children (III.1 and III.2) are neither full nor half-siblings, but are related to some degree in between, sharing an estimated $37.5 \%$ of their genes.

\section{Discussion}

This confirmed case of tetragametic chimerism raises many interesting points to consider while interpreting a purportedly negative DNA paternity test.

One of the most impactful consequences of this case study is to point out that some traditional paternity tests which have resulted in negative outcomes (the tested parent was excluded as the biological parent) may have been wrong, because the alleged parent may have undiagnosed chimerism. It also points to the importance of follow-up testing in such cases. Relatively inexpensive tests are readily available to easily and reliably distinguish between a parent/child and an avuncular relationship.

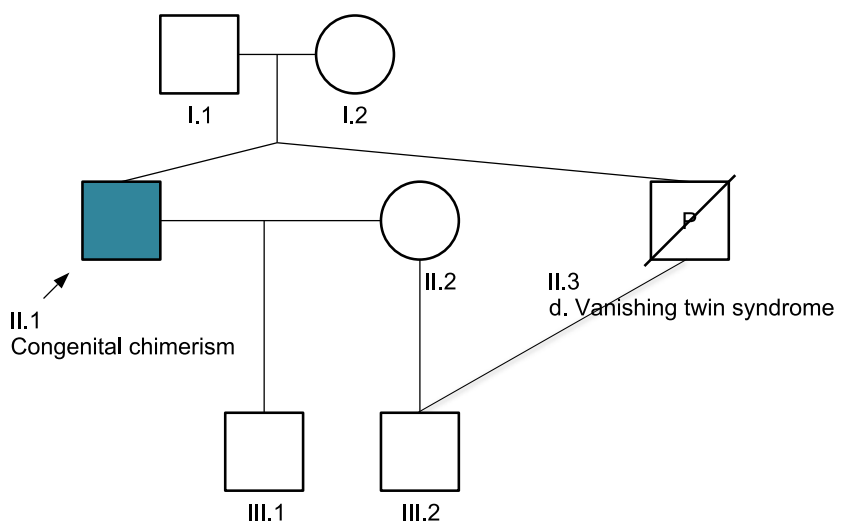

Fig. 2 Proband's pedigree. Tetragametic chimerism gives rise to complex family pedigrees. The proband (II.1) was predicted to have originated as a dizygotic twin pregnancy. Cells from his unborn twin (II.3) were incorporated into his gametes, giving rise to sperm of two distinct cell lines. As a result, the proband's second son (III.2) was conceived via sperm that contained the minor genome, originally from the vanished twin, II.3
Next, we provide recommendations for clinicians and relationship testing labs to prevent future failed parental DNA tests.

\section{Clinical guidance for assisted reproductive technology practitioners}

This unique case presents several guidance points for ART practitioners in terms of evaluating physical findings, interpreting negative parental DNA tests, and facilitating further DNA screening for chimeric individuals.

Most individuals with tetragametic chimerism do not display physical traits related to their condition. However, previously published cases were characterized with one or more of the following manifestations: discordant blood types, patches of different colored hair, different colored eyes, or bicolored skin pigmentation which may display lines of Blaschko (Table 4). Disorders of sexual development (DSDs) can be signs of XX/ $\mathrm{XY}$ chimerism, though most individuals with DSDs will not present with chimerism. It remains unknown as to which traits are most and least prevalent in chimeric individuals.

DNA paternity tests that rely on STR methodologies are likely to be insufficient for undiagnosed chimeric individuals, as they will not necessarily identify avuncular relationships. Moreover these tests may yield different outcomes, depending on the origin of the DNA samples and on the technology utilized. Had the proband only pursued standard DNA paternity testing, he would have had to concede to a false negative result, with tremendous ramifications for him and for his family.

In the event of a purported failed paternity test, it may be in everyone's interests for the fertility clinic to determine whether the father is an uncle or unrelated to the child using a microarray-based DNA test as was used in this case. Refer to Fig. 1 as an example of SNP-based microarray results. These tests can simply and reliably identify the avuncular relationships which can result from a parent with tetragametic chimerism.

Table 4 Physical traits associated with chimerism. Most individuals with chimerism do not manifest physical traits indicative of their condition, and as a result, are believed to remain undiagnosed. Previous cases have described one or more physical traits listed above, which are associated with the phenotype of blood-exclusive or tetragametic chimerism

\begin{tabular}{ll}
\hline Physical trait & Type of chimerism \\
\hline Discordant blood types & $\begin{array}{c}\text { Blood-exclusive } \\
\text { or tetragametic } \\
\text { Patchy, different colored hair }\end{array}$ \\
$\begin{array}{l}\text { Tetragametic } \\
\text { Bifferent colored eyes } \\
\quad \text { (depicting lines of Blaschko) }\end{array}$ & Tetragametic \\
Disorders of sexual development & Tetragametic \\
\hline
\end{tabular}


If the man and the child appear unrelated via the microarray-based test, then paternity issues and/or fertility clinic mistakes should be followed up on. If, on the other hand, the test shows an avuncular relationship, then the parents and/or clinic may want to consider additional testing to determine if the father is indeed a chimera. Given the complexity of the situation and the rapidly changing landscape of genetic/genomic testing, we suggest contacting a genetics expert for advice regarding follow-up testing options.

\section{Technological and logistical test limitations}

The current gold standard for paternity testing involves querying 15 to 20 STRs, using simple PCR technology developed in the late 1980s. Most labs prefer buccal samples, and not all labs may accept semen samples, which would be required in the case of male chimeras. STR-based testing of semen DNA can also fail due to insufficient sensitivity of the test, if the minor genome is rare (less than $\sim 5 \%$ ), in that tissue sample.

Interestingly, one exception is with non-invasive prenatal paternity testing, which utilizes SNP-based microarray on a sample of the mother's blood. This test, we surmise, may have similar detection rates to ancestry testing if the chimeric parent's minor genome is present at detectable levels in cell-free fetal DNA and if the lab reports second degree relationships.

Diagnosing a mother with tetragametic chimerism may be substantially more difficult, especially if the minor genome is not present in cervical cells or cells from other easily accessible tissues. Two highly publicized cases involving mothers with tetragametic chimerism, Karen Keegan and Lydia Fairchild, required additional molecular genetic testing to confirm their diagnoses and resolve issues surrounding maternity. Keegan's condition was diagnosed by extracting DNA from a thyroid tissue sample [11], whereas Fairchild's condition was diagnosed through DNA derived from a cervical test [12]. For females, procuring and testing gametes may involve substantially more complex processes.

SNP-based microarrays also have limitations for conclusively identifying someone as a chimera. The lower limits of detection of chimeras using this particular SNP platform are currently unknown. We believe the sensitivity of SNP-based arrays in detection of tetragametic chimerism is similar to that of somatic mosaicism, if not better. Conlin et al. were able to detect a chimeric XX/XY individual using SNP-array technology. The sample was estimated to have between 20 and $45 \%$ chimeric cells present. Conlin et al. predict SNP-based assays to be capable of detecting samples with as low as 5\% chimerism [13].

Genetic testing is evolving rapidly. Costs of more robust platforms such as Next Generation Sequencing are dropping equally quickly. In the near future, single cell sequencing may become more affordable and accessible to parental DNA testing clients who require follow-up testing. For additional current information, we recommend contacting a genetic specialist with expertise in DNA relationship testing.

\section{DNA relationship laboratory test reporting and legal recommendations}

Considering the serious implications of false negative paternity results, there is a need for a more rigorous system that anticipates chimeras and provides safeguards against false negative test reporting. Currently, the American Association of Blood Banks (AABB) Relationship Testing Program Unit does not specify recommendations about chimeras for laboratories that offer relationship DNA testing in the USA. Although the AABB does establish thresholds, cutoffs for inclusion/exclusion differ from laboratory to laboratory [1], interpretation may be subjective, human errors are possible, and genetic anomalies could impact the conclusions.

We recommend that relationship DNA testing laboratories inform clients of the residual risk of false negative results due to genetic inconsistencies such as chimerism, ideally during informed consent, but certainly on any report that excludes the tested parent. Clients should be made aware that differing results may arise from different tissue samples. Clients need to be educated about inconsistencies, preferably during the process of informed consent. This way, they can be aware of when to pursue further help in case they get an unexpected negative (exclusive) result. Relationship DNA testing labs may consider running tests that assess residual Combined Paternity Index (CPI) values and annotate reports with a pattern suggestive of avuncular relationships. This information would help identify possible chimerism in the tested father and avoid false paternity exclusions resulting from undiagnosed chimerism.

If a couple disagrees with a negative paternity result of a DNA test, they may want to consider contacting a genetic expert specializing in DNA paternity testing for additional testing options.

Legal representatives that utilize relationship DNA tests need to be made aware of limitations and the differences in DNA testing methodologies. They should also be provided with guidelines for next steps if inconsistencies are suspected. [14]

In summary, a PCR-based paternity test that yields a negative result may be insufficiently sensitive to identify the second degree relationship that can occur between a parent with tetragametic chimerism and his or her child. Follow-up testing using microarray-based relationship tests and consultation with a genetics expert could help the clinic and parents to correctly identify the relationship and preserve parental rights.

\section{Future research}

Understanding the prevalence of tetragametic chimerism can both be a study with its own academic merit and a data that 
would highlight the likelihood of missed paternity. Future research into large databases of SNP-microarray data and other highly sensitive DNA testing could provide a window into the prevalence within single tissue types. Single tissue tests, however, would not be sufficient in excluding chimerism from an individual, as the minor genome may be present only in other tissues. Still, research in that direction could be a good starting point to shed more light on the prevalence of chimerism.

Parental and forensic DNA testing labs might revisit cases received that were inconclusive due to contamination. Utilizing different testing methodologies, these cases may yield chimeric individuals.

Further studies of human mosaicism are warranted. Cases involving mosaicism, most notably of sex chromosomes (XX/ $\mathrm{XY}$ ), may in fact be undiagnosed chimerism.

A relevant study of chimerism to consider would be the collection of data on the prevalence of more than one genome in semen. Such a study would shed more light on the prevalence of chimerism as it pertains to paternity.

Better understanding of the detection limits of STR, SNPmicroarrays and other DNA tests utilized in diagnosing and detecting chimerism is needed.

The full psychosocial impact of a diagnosis of chimerism has on the individual and their family members could not been formally studied and is another area that could benefit from research.

Acknowledgments We are grateful to the proband and family for sharing their story and for involving us in their quest for the truth. We would like to acknowledge DNA Diagnostics Center for performing all confirmatory testing and associated interpretations pro-bono for this case.

\section{Compliance with ethical standards}

Conflict of interest The authors declare that they have no conflicts of interest.

Open Access This article is distributed under the terms of the Creative Commons Attribution 4.0 International License (http:// creativecommons.org/licenses/by/4.0/), which permits unrestricted use, distribution, and reproduction in any medium, provided you give appropriate credit to the original author(s) and the source, provide a link to the Creative Commons license, and indicate if changes were made.

\section{References}

1. Annual report summary for testing. Relationship Testing Program Unit; American Association of Blood Banks Annual Report; 2010.

2. Gunn PR, Trueman K, Stapleton P, Klarkowski DB. DNA analysis in disputed parentage: the occurrence of two apparently false exclusions of paternity, both at short tandem repeat (STR) loci, in the one child. Electrophoresis. 1997;18:1650-2.

3. Boklage CE. Embryogenesis of chimeras, twins and anterior midline asymmetries. Hum Reprod Oxf Engl. 2006;21:579-91.

4. Wolinsky H. A mythical beast. Increased attention highlights the hidden wonders of chimeras. EMBO Rep. 2007;8:212-4.

5. Gammill HS, Nelson JL. Naturally acquired microchimerism. Int J Dev Biol. 2010;54:531-43.

6. Vymetalova Y, Bohuslavova R, Hubacek JA, Dufkova B, Kocik M, Malek I, et al. High prevalence of microchimerism in female patients. Transplant Proc. 2008;40:3685-7.

7. Malan V, Vekemans M, Turleau C. Chimera and other fertilization errors. Clin Genet. 2006;70:363-73.

8. Boklage CE. Chapter 4: The frequency and survivability of natural twin conceptions. Mult. Pregnancy Epidemiol. Gestation Perinat. Outcome [Internet]. 1st ed. New York: Taylor and Francis Group.; [cited 2017 Jul 21]. p. 41-2, 49. Available from: https://www. lehmanns.de/shop/medizin-pharmazie/583671-9781850706663multiple-pregnancy

9. Loriaux A, Boulet S, Delorme V, Althuser M, Giroud Lathuile C, Grego S, et al. Tetragametic chimerism: case report. J Gynecol Obstet Biol Reprod (Paris). 2011;40:77-80.

10. Miura K, Niikawa N. Do monochorionic dizygotic twins increase after pregnancy by assisted reproductive technology? J Hum Genet. 2005;50:1-6.

11. News ABC. She's her own twin [Internet]. ABC News. 2014 [cited 2017 Jul 21]. Available from: http://abcnews.go.com/Primetime/ shes-twin/story?id=2315693

12. Yu N, Krushall M, Yunis J. Disputed maternity leading to identification of tetragametic chimerism. N Engl J Med. 2002:1545-52.

13. Conlin LK, Thiel BD, Bonnemann CG, Medne L, Ernst LM, Zackai EH, et al. Mechanisms of mosaicism, chimerism and uniparental disomy identified by single nucleotide polymorphism array analysis. Hum Mol Genet. 2010;19:1263-75.

14. Sheets KM, Baird M, Berger D. Navigating DNA testing in immigration cases. Am. Immigr. Lawyers Assoc. 2015;13. Also Published in Lexis: Bender's Immigration Bull. 2016;22:719-26. 